\title{
Transischiorectal fossa approach for resection of pudendal nerve schwannoma: case report
}

\author{
Stephanie Chen, MD, Brandon Gaynor, MD, and Allan D. Levi, MD, PhD \\ Department of Neurological Surgery and the Miami Project to Cure Paralysis, University of Miami Miller School of Medicine, \\ Miami, Florida
}

\begin{abstract}
Pudendal nerve schwannomas are very rare, with only two cases reported in the English-language literature. The surgical approaches described in these two case reports are the transgluteal approach and the laparoscopic approach. The authors present the case of a patient with progressive pelvic pain radiating ipsilaterally into her groin, vagina, and rectum, who was subsequently found to have a pudendal schwannoma. The authors used a transischiorectal fossa approach and intraoperative electrophysiological monitoring and successfully excised the tumor. This approach has the advantage of direct access to Alcock's canal with minimal disruption of the pelvic muscles and ligaments. The patient experienced complete relief of her pelvic pain after the procedure.
\end{abstract}

http://thejns.org/doi/abs/10.3171/2016.4.SPINE151449

KEY WORDS schwannoma; pudendal nerve; ischiorectal; technique

$\mathrm{P}$ ELVIC schwannomas are rare tumors, with only two previously reported cases of pudendal nerve schwannomas in the English-language literature. ${ }^{4,8}$ However, entrapment of the pudendal nerve in Alcock's canal is a well-described phenomenon. This syndrome is characterized by perineal and vulvar or scrotal pain that worsens with sitting. ${ }^{2}$ Similar surgical approaches for release of pudendal nerve entrapment and pelvic schwannomas have been described. Those used in the two case reports of pudendal nerve schwannomas were the transgluteal approach and laparoscopic approach.

We present the first report of a pudendal nerve schwannoma excision via transischiorectal approach with ultrasound assistance.

\section{Case Report}

History and Examination

A 43-year-old woman presented with a 3-year history of intermittent stabbing pain referred to the vagina and rectum. The pain was accompanied by a sensation of fullness and pressure in the rectum. Symptoms progressed in frequency over the years and she also began experiencing right-sided pelvic pain radiating into her right gluteus and medial thigh. Her pain was worse in the sitting position. Her medical history was significant only for a benign cardiac arrhythmia. Previous surgeries included breast augmentation, right anterior cruciate ligament repair, ulnar nerve transposition, and multiple cervical spine surgeries. Diagnostic workup included abdominal CT revealing a $2.4-\mathrm{cm}$ mass in the right ischiorectal fossa (Fig. 1) and MRI showing heterogeneous enhancement of the lesion (Fig. 2). A CT-guided biopsy prior to referral was performed with pathology suggestive of schwannoma. On examination, patient's abdomen was soft and nontender. Rectal tone and perineal sensation were intact. The remaining findings on her neurological examination were normal. Surgical management was recommended due to the size of the tumor and progression of symptoms attributed to the mass.

\section{Operation}

The tumor was difficult to palpate given the thick overlying adipose tissue (Fig. 3); thus, on the day of surgery, the patient underwent transgluteal ultrasound for localization and planning of the incision. A vertical incision was planned to avoid the gluteal cleft. Avoidance of this cleft is of paramount importance in minimizing the risk of injury to the anus, thus also decreasing risk of complications including infection, poor wound healing, and fistulous tracts. The patient was then taken to the operating room and general anesthesia was induced. She was positioned prone on 


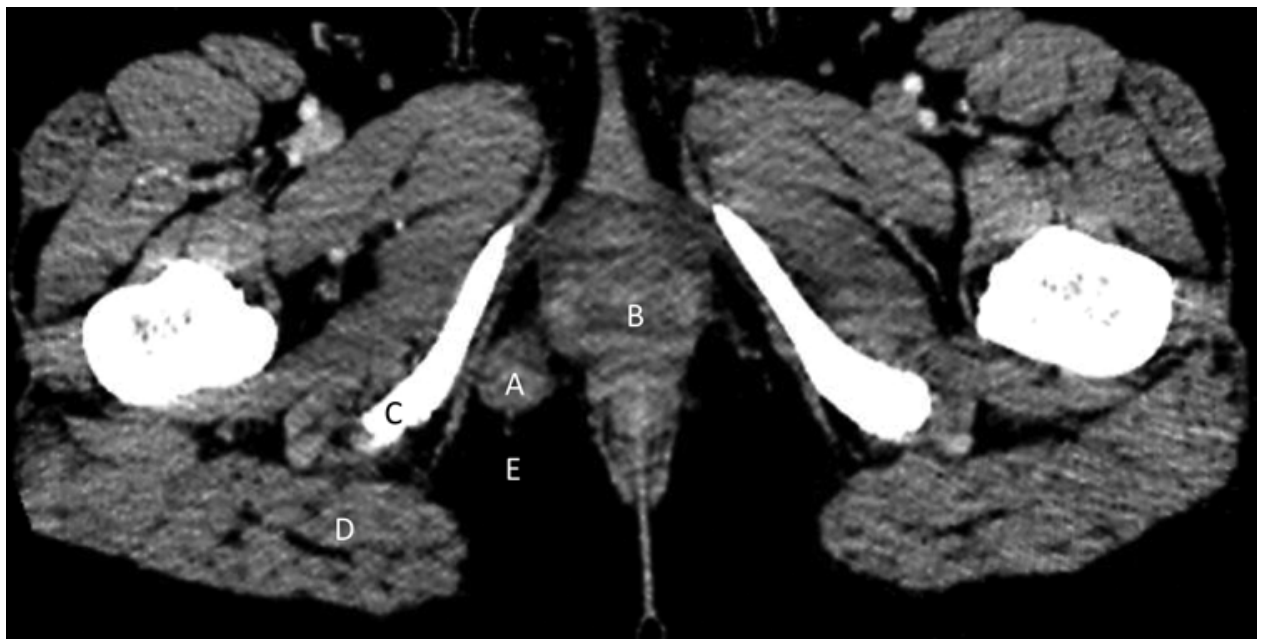

FIG. 1. Axial contrast-enhanced CT scan of the pelvis demonstrating an isointense mass arising from the pudendal nerve: pudendal nerve schwannoma $(A)$, rectum $(B)$, ischial tuberosity $(C)$, gluteus maximus muscle $(D)$, and ischiorectal fossa $(E)$.

gel rolls and the operating table was placed in a jackknife position. The right gluteal region was prepared and draped in a sterile fashion.

A vertical incision was made in the right infragluteal region lateral to the anus and rostral to the ischial tuberosity. Subcutaneous fat was dissected and the mass was exposed with sharp dissection and microscopic magnification just medial to the ischial tuberosity which could be easily palpated. Intraoperative nerve stimulation was used to verify pudendal innervation with strong electromyography (EMG) contraction of the rectal sphincter observed. Within Alcock's canal the tumor was seen arising from a branch of the pudendal nerve that did not elicit motor contraction. We incised the true capsule of the tumor and used sharp dissection to resect the tumor. Intraoperative frozen section pathology was consistent with schwannoma. Nerve stimulation at the end of the case showed normal electrophysiological responses from the anal sphincter.

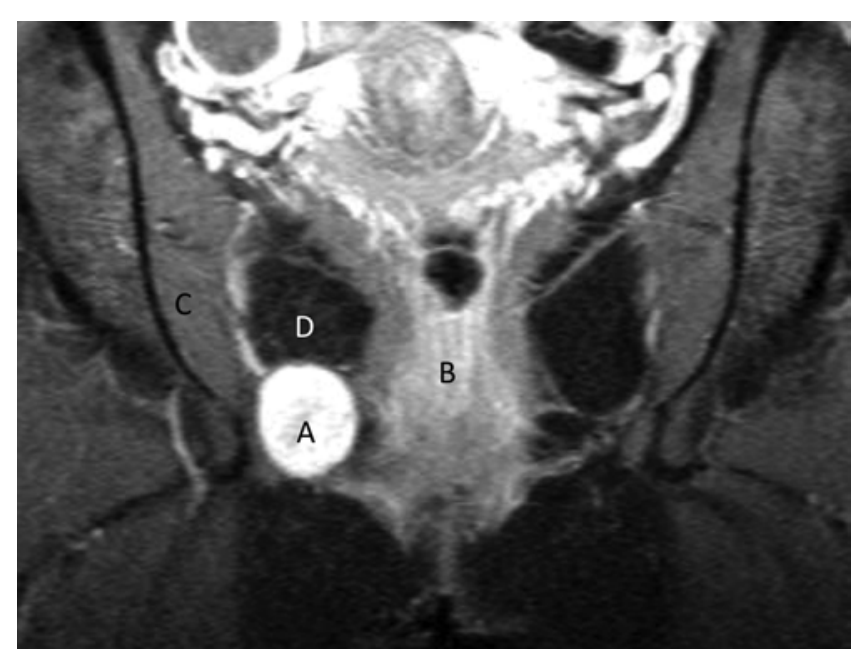

FIG. 2. Coronal T1-weighted, fat-suppressed Gd-enhanced MR image demonstrating intense heterogeneous contrast enhancement of the pudendal nerve schwannoma: pudendal schwannoma $(A)$, rectum $(B)$, obturator internus muscle $(C)$, and ischiorectal fossa $(D)$.
Hemostasis was achieved with bipolar electrocautery and the wound was copiously irrigated. A Hemovac drain was placed in the surgical bed and removed on the 1st postoperative day.

\section{Postoperative Course}

The patient was discharged the day after surgery and had an uneventful recovery.

The final pathology was a $2.2 \times 2.0 \times 2.0-\mathrm{cm}$, wellencapsulated, well-circumscribed, pale-tan, brown softtissue mass. Sectioning revealed areas of hemorrhage and a spindle cell pattern consistent with schwannoma.

At the neurosurgery follow-up appointments 1 week and 3 months later, the patient described complete resolution of her perineal pain. She did not experience any numbness or bowel or bladder incontinence.

\section{Discussion}

The pudendal nerve arises from the S-2, S-3, and S-4 sacral nerve roots proximal to the sacrospinous ligament. The nerve courses between the piriformis and coccygeus muscles and exits the pelvis through the greater sciatic foramen. It then crosses behind the sacrospinous ligament and reenters the pelvis through the lesser sciatic foramen. Within the pelvis the pudendal nerve, as well as internal pudendal artery and vein, travels forward along the lateral wall of the pelvis wrapped inside the sheath of the obturator internus fascia known as Alcock's canal (pudendal canal). Inside Alcock's canal the nerve divides into the inferior rectal nerve, the perineal nerve, and the dorsal nerve of the penis or clitoris. ${ }^{3,6}$

The pudendal nerve has both motor and sensory functions. It is responsible for sensation of the perineum teguments (glans penis, clitoris, scrotum, labia majora, skin of the central fibrous perineal body, and anus) and controls motor function for sexual arousal, micturition, and defecation. ${ }^{6}$

Schwannomas are benign nerve sheath tumors com- 


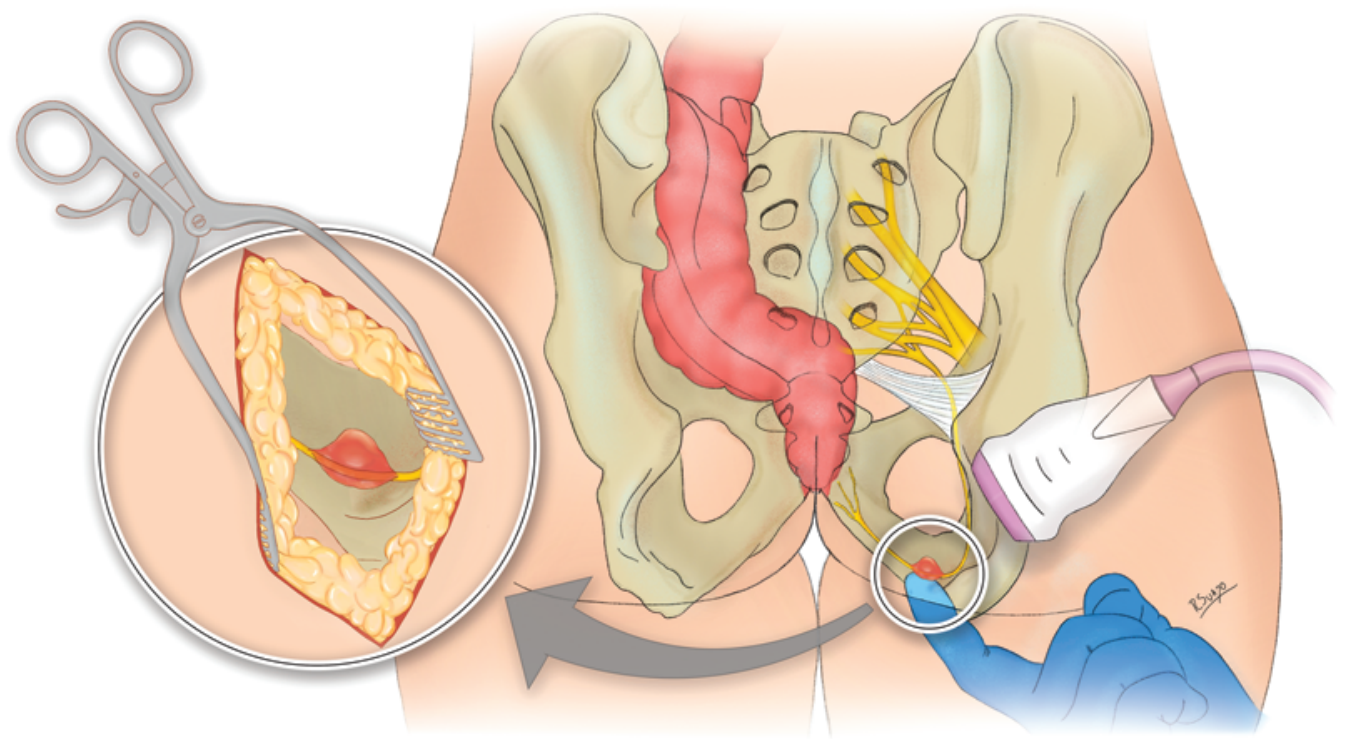

FIG. 3. Diagram demonstrating the pudendal nerve arising from the S2-4 nerve roots, exiting through the greater sciatic foramen, coursing behind the sacrospinous ligament, reentering the pelvis through the lesser sciatic foramen, and coursing along Alcock's canal. The pudendal nerve schwannoma was located where the nerve exits Alcock's canal. Preincisional ultrasound was used to help localize the incision. Inset: A higher-power view of the relationship of the tumor to the pudendal nerve. Copyright University of Miami Miller School of Medicine. Published with permission.

posed entirely of neoplastic Schwann cells. ${ }^{7}$ In a series from Kim et al., only 6 (4.8\%) of 124 schwannomas were located in the pelvic region. ${ }^{1}$

The primary treatment is excision with preservation of the parent nerve. Schwannomas typically arise from a single nonfunctioning sensory fascicle. However, given the rarity of pudendal nerve schwannomas, the anatomical landmarks and operative technique are relatively unfamiliar to neurosurgeons. In this case report, we describe the various approaches that have been described to access the pudendal nerve within the literature.

A laparoscopic approach has been reported for retroperitoneal tumors. Mazzola et al. described a case of successful resection of a pudendal nerve schwannoma using laparoscopy. ${ }^{4}$ In this approach, 3 ports are placed near the umbilicus and dissection is performed along the iliac fossa, incising the peritoneum over the umbilical ligament and down through the endopelvic fascia. However, this technique is limited by the size of the tumor and its adherence to vital vessels.

Alternatively, a transgluteal approach has been described for both pudendal nerve entrapment syndrome and a pudendal nerve schwannoma. ${ }^{5,8}$ This approach accesses the pudendal nerve where it crosses posterior to the sacrospinous ligament. The gluteus maximus muscles and underlying gluteus fascia are opened, the sacrotuberous ligament is transected, and the pudendal nerve can be found just posterior to the sacrotuberous ligament in the ischiorectal fossa adipose tissue..$^{5}$

The most appropriate operative technique for a pudendal schwannoma depends on the location of the tumor. The transischiorectal fossa approach is ideal for tumors located in the ischiorectal fossa, near Alcock's canal. This approach has the advantages of direct access to the tumor without disrupting any of the pelvic ligaments or muscles, thus minimizing complications and recovery time.

Intraoperative electrophysiological monitoring of the pudendal nerve using the anal sphincter is also simple and effective. It can aid in identifying the nerve of origin and ensure preservation of nerve responses when dissecting the tumor off of the nerve. Somatosensory evoked potential monitoring can also be considered, but, given the small size of the sensory branches supplying the perineal region, the recordings are technically more difficult than the EMG recordings of the anal sphincter muscle.

\section{Conclusions}

Pudendal nerve schwannomas are a rare entity. The transischiorectal fossa approach after preoperative ultrasound localization is safe and effective to achieve a grosstotal resection.

\section{Acknowledgments}

Dr. Levi receives grant support from the Department of Defense.

\section{References}

1. Kim DH, Murovic JA, Tiel RL, Moes G, Kline DG: A series of 397 peripheral neural sheath tumors: 30-year experience at Louisiana State University Health Sciences Center. J Neurosurg 102:246-255, 2005

2. Labat JJ, Riant T, Robert R, Amarenco G, Lefaucheur JP, Rigaud J: Diagnostic criteria for pudendal neuralgia by pudendal nerve entrapment (Nantes criteria). Neurourol Urodyn 27:306-310, 2008

3. Maldonado PA, Chin K, Garcia AA, Corton MM: Anatomic variations of pudendal nerve within pelvis and pudendal 
canal: clinical applications. Am J Obstet Gynecol 213:727. e1-727.e6, 2015

4. Mazzola CR, Power N, Bilsky MH, Robert R, Guillonneau B: Pudendal schwannoma: a case report and literature review. Can Urol Assoc J 8:E199-E203, 2014

5. Peltier J: Anatomical basis of transgluteal approach for pudendal neuralgia and operative technique. Surg Radiol Anat 35:609-614, 2013

6. Robert R, Labat JJ, Riant T, Louppe JM, Hamel O: [The pudendal nerve: clinical and therapeutic morphogenesis, anatomy, and physiopathology.] Neurochirurgie 55:463-469, 2009 (Fr)

7. Skovronsky DM, Oberholtzer JC: Pathologic classification of peripheral nerve tumors. Neurosurg Clin N Am 15:157-166, 2004

8. Tollstrup T, Dellon AL: Schwannoma of the pudendal nerve at sciatic notch and differential diagnosis of "sitting pain." J Reconstr Microsurg 30:283-286, 2014

\section{Disclosures}

Dr. Levi has received teaching honoraria from Medtronic Sofamor Danek.

\section{Author Contributions}

Conception and design: all authors. Acquisition of data: all authors. Analysis and interpretation of data: all authors. Drafting the article: all authors. Critically revising the article: all authors. Reviewed submitted version of manuscript: all authors. Approved the final version of the manuscript on behalf of all authors: Levi. Study supervision: Levi.

\section{Correspondence}

Allan D. Levi, Department of Neurological Surgery, University of Miami Miller School of Medicine, Lois Pope LIFE Center, 1095 NW 14th Terrace (D4-6), Miami, FL 33136. email: alevi@ med.miami.edu. 